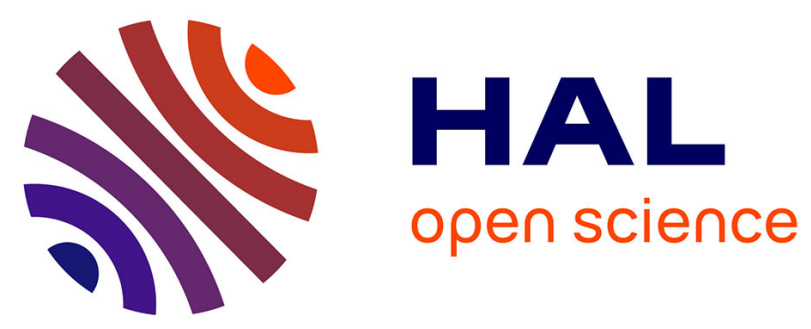

\title{
An alternative approach to interferogram collection and processing for a vintage Bomem DA3 Fourier transform spectrometer
}

B.G. Guislain, R.A.R. Harvey, Dennis W. Tokaryk, Amanda J. Ross, P. Crozet, A.G. Adam

\section{To cite this version:}

B.G. Guislain, R.A.R. Harvey, Dennis W. Tokaryk, Amanda J. Ross, P. Crozet, et al.. An alternative approach to interferogram collection and processing for a vintage Bomem DA3 Fourier transform spectrometer. Journal of Molecular Spectroscopy, 2019, 364, pp.111181. 10.1016/j.jms.2019.111181 . hal-02282973

\section{HAL Id: hal-02282973 \\ https://hal.science/hal-02282973}

Submitted on 28 Oct 2020

HAL is a multi-disciplinary open access archive for the deposit and dissemination of scientific research documents, whether they are published or not. The documents may come from teaching and research institutions in France or abroad, or from public or private research centers.
L'archive ouverte pluridisciplinaire HAL, est destinée au dépôt et à la diffusion de documents scientifiques de niveau recherche, publiés ou non, émanant des établissements d'enseignement et de recherche français ou étrangers, des laboratoires publics ou privés. 


\title{
An alternative approach to interferogram collection and processing for a vintage Bomem DA3 Fourier transform spectrometer
}

\author{
B. G. Guislain, R. A. R. Harvey, D. W. Tokaryk* \\ Physics Department and Centre for Laser, Atomic, and Molecular Sciences, University \\ of New Brunswick, Fredericton, NB, Canada E3B 5 A3 \\ A. J. Ross, P. Crozet \\ Institut Lumière Matière, UMR5306 Université Lyon 1-CNRS, Université de Lyon, \\ 69622 Villeurbanne cedex, France
}

A. G. Adam

Chemistry Department and Centre for Laser, Atomic, and Molecular Sciences, University of New Brunswick, Fredericton, NB, Canada E3B 5A3

\begin{abstract}
We describe a new way of collecting data from Bomem DA3 Fourier transform spectrometers, bypassing the original computers used by these instruments, which are obsolete and more prone to failure than other parts of the system. We collect the interferogram, helium-neon reference laser, and a trigger marking zero path difference with a modern computer as a function of time at a high data rate, as opposed to as a function of position along the scanning arm of the interferometer. The interferogram is then reconstructed as
\end{abstract}

\footnotetext{
* Corresponding author
}

Email addresses: dtokaryk@unb.ca (D. W. Tokaryk), amanda.ross@univ-lyon1.fr (A. J. Ross), patrick.crozet@univ-lyon1.fr (P. Crozet), agadam@unb.ca (A. G. Adam) 
a function of position (measured in helium-neon fringes) by interpolation. The resulting spectra are at least as good as those obtained from the original data processing methods. This work can be used as a model for reviving other DA3 systems which are in good order with respect to mechanical and electronic components, but with nonfunctional computers, or for collecting and processing data for any other Fourier transform spectrometers.

Keywords: Bomem DA3, Fourier transform spectroscopy, metal hydride, laser induced fluorescence 


\section{Introduction}

Bomem Inc of Québec, Canada was the first company to commercialize high-resolution Fourier transform spectrometers, in the early 1980s[1]. These instruments allowed spectral retrieval over a very wide region, from about $10-40000 \mathrm{~cm}^{-1}$, at the impressive ultimate resolution of $0.0025 \mathrm{~cm}^{-1}$, and with resolvance of $10^{6}$ at any wavelength. The tube containing the scanning mirror of the Michelson interfometer was mounted vertically to minimize the floorspace that the spectrometer occupied. The tube, spectrometer body, and compartment containing samples and detectors were evacuable to minimize absorption from atmospheric gases. The mechanical subsystems and the simple electronics and on-board microprocessors that operated them have proven robust, and some of the DA3 units around the world are still functioning despite their near-40-year age.

However, the computers originally used to control the DA3s and to process the data, proved to be far more fragile and prone to obsolescence. The earliest versions of the spectrometers used PDP-11 computers that managed a custom-built 'vector processor', which had 500 kilobytes of random-access memory to collect, store and coadd the interferograms, and to transform them into spectra. Upgrades to VAX, and then Windows-based systems were progressively introduced, and in the late 1990s an upgrade called the PCDA was offered, running on a Pentium-based computer with 1 megabyte of memory to improve the data collection and processing. Several of the original DA3 on-board electronic cards were replaced to implement the PCDA. Bomem discontinued the DA3 line in 2000, and users of the instruments have maintained legacy computer systems to run them, with the added problem 
of finding obsolete electronic components to repair inevitable failures. Over the years, some third-party vendors have attempted to fill this niche by producing alternative interfaces, some of which are completely independent of Bomem computer systems while others provide new control for original vector processor units.

The genesis of this paper lies in two Bomem DA3.002 systems, one now housed in the molecular spectroscopy laboratory at the University of New Brunswick (UNB) in Canada, and the other at Université de Lyon in France, at the Institut Lumière Matière (ILM). The UNB instrument was originally located at the University of British Columbia, and was used extensively by prominent researchers in molecular spectroscopy including Anthony J. Merer, Michael C. L. Gerry and Irving Ozier. The instrument was moved to UNB when its group of principal users at UBC retired, but because its vector processor unit did not survive transit, it has remained dormant for several years. The ILM instrument has been in continuous use since 1989, primarily for recording visible and near-infrared laser-induced fluorescence (LIF) from small radical molecules. This DA3 was upgraded in 1999 with a PCDA system, and although it is still functional, its computers face the problems related to obsolescence described above.

The UNB and ILM groups collaborate on projects of mutual interest, and make frequent visits to each other's laboratories. During one of these visits in the summer of 2017, we discussed the possibility that the UNB instrument might be revived, if the interferograms and associated data it produces could be acquired by passively capturing data streams as the spectrometer was run in 'test mode', in which it continuously scans without computer control. We 
confirmed that the interferometer per se was still functional, retaining alignment and providing analog output from photodiodes detecting signals from a helium-neon (He-Ne) reference laser, a broadband incandescent bulb used in alignment, and the data itself. In the summer of 2018, we implemented our ideas using the still-functional Bomem system at ILM, which afforded us some comparison between the original data collection procedure and our new method, and then successfully employed the method to the UNB instrument. We note that the wealth of information provided in the Bomem system manual was key to our success.

The rest of this paper describes the process, considerations and results of developing a new way to operate the DA3s both in Lyon and at UNB. We assume that the reader is familiar with the basic principles of Fourier transform spectroscopy, and we will focus on the specific implementation of those principles in the DA3 system.

\section{The principles}

In order to produce a spectrum from a Fourier transform interferometer, one must

1. sample the interferogram associated with a light source that is being modulated by the Michelson interferometer as its moving mirror is scanned, densely enough to fully capture the fringe pattern without aliasing,

2. accurately determine the moving mirror position during the scan at each sample of the interferogram, and 
3. assess the moving mirror position at which the path difference between light traveling along the two arms of the interferometer is zero.

The Bomem DA3 provides the analog signal from the detector at a BNC connector labelled 'SIG OUT' on the data connector card on the top of the instrument's main body, so the interferogram can be collected and digitized easily. Measurement of the scanning mirror's position is achieved by recording the interference from a single-mode He-Ne laser signal which is modulated in the interferometer simultaneously with light undergoing analysis. This signal is a relatively uniform sinusoidal ripple, and is available by tapping into the ' $\mathrm{R}$ ' test point on an alignment card mounted to the back of the DA3. The zero-path-difference (ZPD) position is determined by recording the signal from a small broadband incandescent lamp also mounted to the back of the DA3. The lamp signal is also modulated by the interferometer, and is detected by sensors on the same alignment card that detects the He-Ne laser signal. The interferogram from the broadband lamp exhibits a fringe cluster of large amplitude near ZPD, since at this position the phase difference between light waves traversing the two arms of the interferometer is zero and the light experiences constructive interference for all frequencies. This ZPD fringe cluster is unambiguous, and dominates the interferogram. The lamp's interferogram can be digitized directly from the 'ZPD' test point on the alignment card, but in conventional operation a voltage level is set on the card so that when the interferogram's amplitude increases above this threshold a TTL signal changes state from low to high. The TTL signal is available on the top of the instrument from a BNC labeled 'SYNC ZPD', near to the 'SIG OUT' connector. 
The original design of the Bomem data collection electronics and software relied upon electronic detection of a trigger point within a fringe cycle of the He-Ne reference signal to mark when analog-to-digital (A/D) conversions of the interferogram were to occur. The system could record signals every $N$ triggers for sampling interferograms of long-wavelength light, and could be subdivided through real-time interpolation into 2, 4 or 8 samples between triggers. All of these schemes produced sampling at well-defined distances along the tube, given by either multiples or integer fractions of the He-Ne wavelength. This was not convenient for us to implement, so we proposed to collect the data as a function of time, and convert the data streams to functions of the scanning mirror position in software post-collection.

\section{Implementation}

Compared to the early 1980s when the DA3 system was introduced, a contemporary scientist has some enormous advantages: a current-generation computer's calculational capability dwarfs what was available nearly 40 years ago, and both gigabytes of RAM and terabytes of hard drive space are cheap and readily available. The original Bomem software sampled data as judiciously as possible, exploiting aliasing to reduce the number of points required to minimally sample the signal when its bandwidth was in a narrow range. The transformation algorithms were necessarily limited; the size of the final spectrum could seldom span the full frequency range represented in the data due to memory limitations, and the ability to zero pad the interferogram before transformation was similarly limited, so the number of points defining a spectral line was small. These limits no longer present problems either 
for data sampling or for transformation, and our implementation makes far more generous use of modern data storage and processing capability than was imaginable when the DA3 system was first produced. This is particularly advantageous in the context of broadband emission spectroscopy where band systems can cover several thousand $\mathrm{cm}^{-1}$.

We have used two systems of similar capability to collect signals in the near-infrared and visible spectrum from the FT spectrometer: an ACCES IO USB-AIO16-16E data collection box, and an ADLINK Technology DAQ2006 on-board computer card. The ACCES box can perform 16-bit A/D conversions (matching the original Bomem hardware) at up to $250 \mathrm{kHz}$ if only one channel is used, but its effective maximum rate is only $83.3 \mathrm{kHz}$ when three channels are used. With three channels, each is sampled in turn with a temporal separation of 4 microseconds when the box is asked to make a conversion. The ADLINK card also has 16-bit conversion, but can sample simultaneously at $250 \mathrm{kHz}$ on each of four channels. When collecting data during an experiment, we digitized signals from three sources: the interferogram, the He-Ne fringes and the ZPD SYNC line. The sampling rate was chosen with consideration for the speed at which the moving mirror was scanned, such that about 12-16 data points were taken per He-Ne fringe. For a mirror speed of $0.2 \mathrm{~cm} / \mathrm{s}$, this was achieved with digitization at 80-100 $\mathrm{kHz}$. At this rate, the data are oversampled by a factor of 4 with respect to the sampling algorithm of the original DA3 computers. We note that the ACCES box's hardware creates a slight delay between reading the interferogram, He-Ne and ZPD SYNC signals. Since the He-Ne fringes are only relative markers, this delay is of no consequence for the final spectrum. Sim- 
ilarly, the ZPD signal is used to align and co-add multiple scans, and this function will also be unaffected by the slight delay.

In our earliest implementations, the system was allowed to continuously sample for up to 30 minutes while the spectrometer's moving mirror cycled up and down the scan tube in mode 3 (test mode). The data were either stored in a memory buffer of about 1 gigabyte, then dumped to disk, or in a two-element circular buffer, with one buffer being written to disk while the second was filled. The resulting files were extremely large compared to those collected with a typical Bomem system due to the much higher sampling density employed, and because data were collected as the mirror went up the tube, and while it made a rapid descent back to the bottom. The signals were only useful during the first phase of motion, so some fraction of the stored data was superfluous. Figure 1 shows the ZPD and interferogram signals during one complete cycle of the scanning mirror's motion.

\section{Data processing}

The first stage of processing involved extracting individual scans from the large multi-scan data files. Each scan started when the moving mirror was a few centimeters below the ZPD position, moved through ZPD and then ended at the scan distance appropriate for the desired resolution. The ZPD SYNC line started in a logic 'low' state, changed to high at ZPD, then reverted to low at the end of the scan. It stayed low while the mirror descended, changing briefly to high between ZPD and the return to its initial position, where it once again became low (see Fig. 1). Thus, the ZPD SYNC signal was employed to delimit a scan from the initial position until the end of scan, 

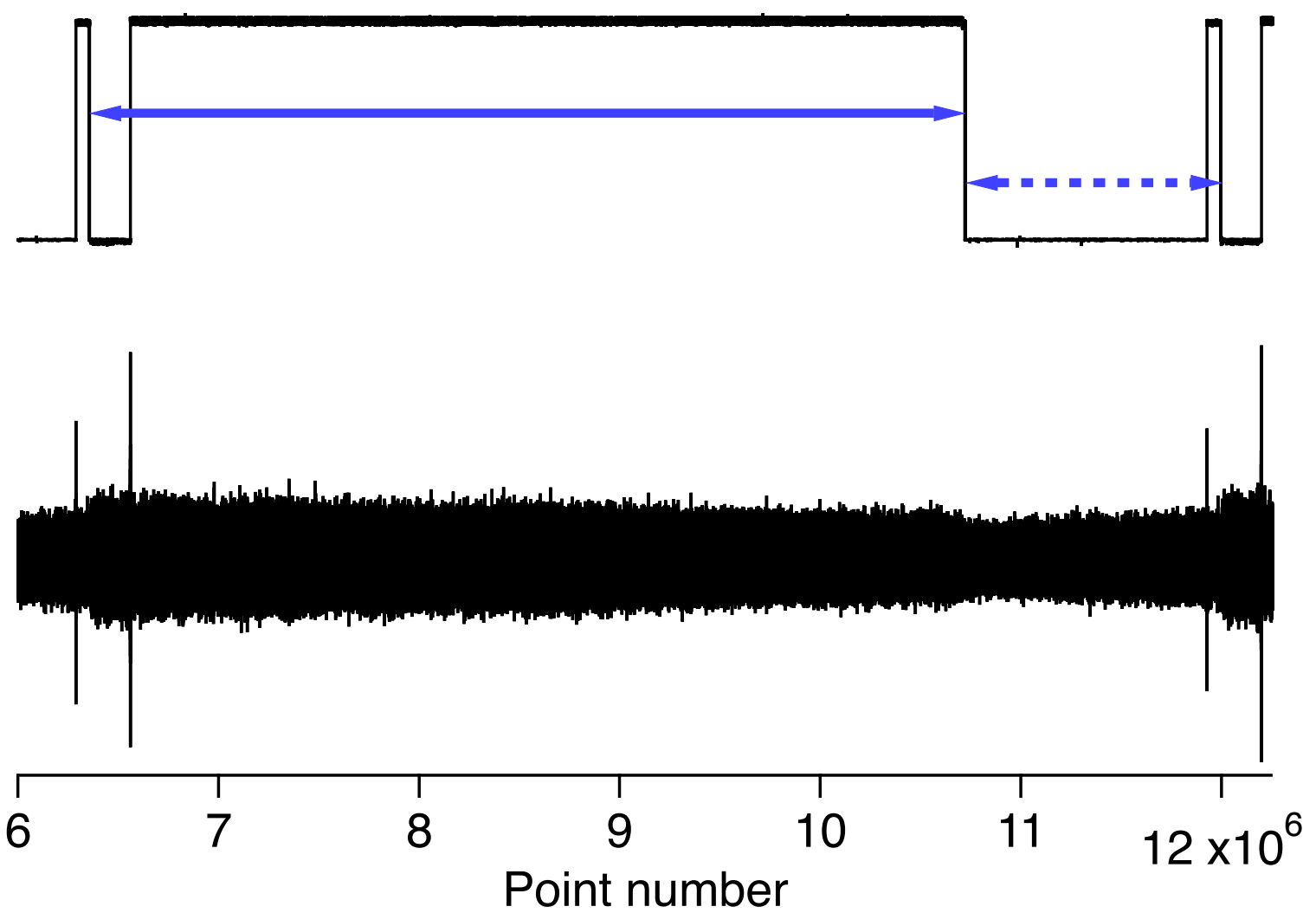

Figure 1: (In colour online.) The ZPD signal (top) and interferogram trace (bottom) over one cycle of the scanning mirror's motion. The sharp features in the interferogram are 'centre bursts' that occur when the scanning mirror passes through the ZPD position. Note that centre bursts occur for both directions of mirror travel, and that the ZPD signal goes high when each burst is recorded, then falls low again at either end of the scan. The solid blue arrow in the ZPD trace marks the range over which data of interest are recorded, while the dotted blue arrow shows the extraneous data collected while the mirror descends. 
based on its changes of state. Data were saved as individual scans between these delimiters.

The abscissa of each scan had to then be converted from time to distance along the scan tube, with the distance origin set to ZPD. Since our distance markers were fringes of the He-Ne laser, we first subtracted the average of this signal from the signal itself, and then sought the pairs of consecutive points between which the signal value crossed zero. ${ }^{1}$ We then linearly interpolated the (noninteger) point value at which the signal value was zero. The halffringe separation given by two consecutive zero crossings was then cut into eight equally-spaced intervals, and the interferogram was interpolated at each of these points and stored. We thus obtained 16 interpolated points of the interferogram per laser fringe. The procedure is valid provided that the motion of the motor moving the scanning mirror was reasonably uniform over a distance of one half-wavelength of the reference laser. The first fringe crossing after the ZPD position was the one to which we assigned an index of zero.

Finally, the individual interferograms were coadded and averaged, with small differences in scan length trimmed as required to ensure that each component making up the coadded interferogram was of equal length. The final interferogram was then phase-corrected and Fourier transformed to produce a final spectrum, using a selection of methods described in section 6 .

\footnotetext{
${ }^{1}$ On occasions when the He-Ne signal's cycle average drifted, the signal was subjected to a 100-point smoothing to remove the fringe oscillations, and the remaining function was subtracted from the unsmoothed data so that zero crossings could still be found and used.
} 


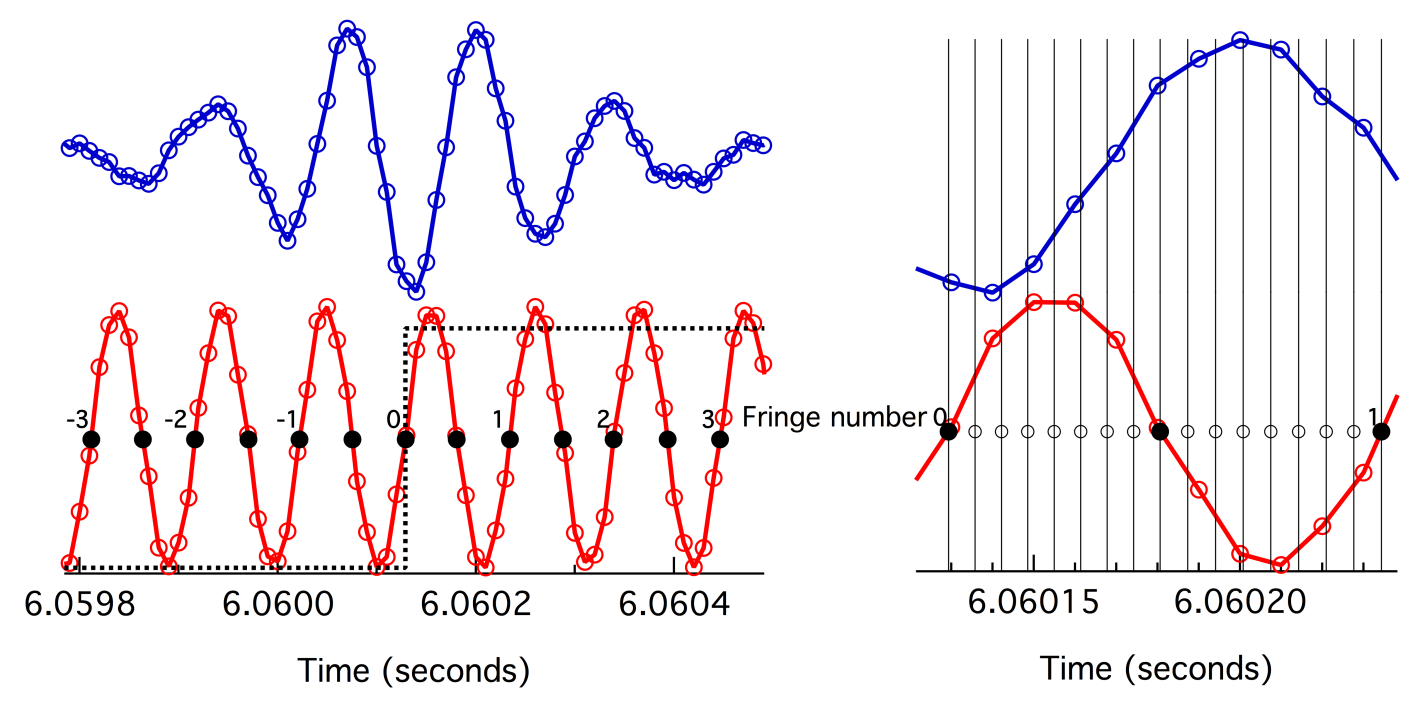

Figure 2: (Colour online) The signals digitized when taking data from the Bomem DA3 with a time-triggered fast data acquisition card. Left: the signals shown are the data interferogram (blue, top waveform), the He-Ne laser fringes (red, bottom waveform), and the ZPD signal (black, dotted line). The open circles represent the sampling points, as a function of time. Black filled circles represent the zero-crossings of the He-Ne fringes, obtained numerically from the sampled fringe signal. Right: the interpolation procedure, in which the signals are interpolated at even increments one eighth of the distance between zero-crossings. The vertical black lines represent the interpolation points, which are used to convert the abscissa from time to fringe position. The absolute fringe number is given on both halves of the figure, with fringe 0 set to the closest zero-crossing to the change in state of the ZPD signal. 


\section{Computer control of the DA3}

The procedure given in section 3 was refined by placing the DA3 unit under computer control. This offered two major advantages. Firstly, each scan could be saved as an individual file with very nearly equal starting and ending points. Disk storage space was reduced since no data were recorded during moving mirror's return to its starting position, and in the event that the spectrometer lost alignment, the scan could be aborted and restarted automatically. Also, a scan could be rejected during processing if it displayed an anomaly; this is not possible if the scans are coadded in memory as they come in. Secondly, while under computer control the spectrometer operates in mode 1 ('remote') or 2 ('local'), in which the mirror's scanning speed is actively stabilized. This results in more even separation between fringes of the He-Ne reference laser.

The control process described in the Bomem system manual was changed for units enhanced with the PCDA upgrade, but almost no documentation was provided in the latter case. We have therefore devised two methods for implementing computer control, depending on whether the system's electronics are original or upgraded.

\subsection{Control of the UNB spectrometer}

The DA3 at UNB has original electronics, and the interfacing protocol is well documented in the Bomem system manual. Data and commands are transferred between the instrument and a computer via a 50-pin connector on

the data connector card. Most of the pins are used for transferring the digitized interferogram values from the spectrometer's internal A/D converter, 
but we have bypassed these in favour of our own A/D converters. Of the remaining lines, three are of interest: the STANDBY line, which places the DA3 in modes $3 / 4$ (independent of the computer) when high or in modes $1 / 2$ (computer control) when low, and the TTX and RRX lines, which allow the computer to communicate with the DA3 via a three-line RS232 serial-port connection.

When computer control of the instrument is initiated with the STANDBY line, the DA3 moves into mode 2 ('local'). If scanning is then initiated, the spectrometer will send single-byte status information when the scanning mirror is at the beginning of a scan, has crossed ZPD, or when the maximum path difference (based on the selected resolution) had been reached, as well as diagnostic and error messages as appropriate. (We monitored these bytes on an oscilloscope to determine the rate of the serial transmission, which was 9600 baud in our case.) Commands can be then sent to set the length and speed of the scan, the number of fringes to collect before ZPD, and many other conditions and parameters. Setting these places the instrument in mode 1 ('remote'), and a command to initiate the run can be given. During the run, software is used for the A/D conversions and to monitor the serial line for status commands. Data are stored in memory or on disk as the scan progresses, and each scan is saved as a separate file, bypassing the need to cut the file into scans as described in section 4. Programming the sequence of events is quite straightforward, given the extensive documentation of the command protocols provided in the Bomem system manual. 


\subsection{Control of the Lyon spectrometer}

The DA3 in Lyon has electronics corresponding to the Bomem PCDA upgrade, with RS422 instead of RS232 communication between the PC and the DA instrument. Not having a compatible interface, we continued to pilot this DA3 with the PCDA software, selecting the option to initiate each scan with an external trigger. Our software, installed on the second computer in which the DAQ2006 card was installed, provided the 'START' signal to the DA3's data connector card to initiate each scan, after which the A/D card collected the three signal channels until the required number of points (as calculated from the scan rate, desired maximum path length and sampling frequency) was reached. The ZPD line was then monitored for a change of state indicating that the scanning mirror had passed this position on its return trip. The scan traces were then written to a file, and after a short delay the cycle was restarted. Data could be taken simultaneously with the PCDA and our new process when running in this manner, which allowed for comparisons between the resulting spectra.

A document has been posted as supplementary material that describes in more detail our implementation of computer control on both the UNB and Lyon DA3 spectrometers.

\section{Performance assessment}

\subsection{Bandwidth and phase correction}

One advantage that arises from our approach is the increase in the available spectral bandwidth due to the increased number of points collected per fringe. Typical scans will sample the He-Ne signal 12-16 times per fringe, 
so the Nyquist limit is pushed into the vacuum-ultraviolet range. Since the instrument is meant to function at considerably longer wavelengths by comparison, concerns regarding aliasing are rendered moot. The increases in computational power and storage compared to the 1980s allow us to collect spectra over the entire bandwidth spanned by the detector used, so one does not need to sacrifice bandwidth for point density or vice versa. We are also free to liberally zero-pad the interferogram to increase the density of points in the final spectrum and to obtain better-defined shapes to the spectral features.

We have so far tested two approaches to phase correction and transformation of the interferogram. The first one, described by Porter et al.[2]), is very convenient to implement. It involves taking a low-resolution phase spectrum, either separately or from a small segment of the full interferogram chosen symmetrically around the centre burst, and fitting it to a linear function with weights taken from the magnitude of the corresponding lowresolution spectrum. The linear function is used to correct the phase of the high-resolution spectrum during transformation. Only one large data set needs to be transformed to generate the final result.

This procedure was effective over smaller bandwidths, but when larger bandwidths were considered the phase function defined in Ref. [2] deviated quite significantly from the linear form $\phi(\omega)=\phi_{0}+\beta \omega$ employed in the theoretical development. The omission of higher-order terms caused distortion in the shapes of spectral lines near the edges of the spectrum. This happens to be a serious issue for our applications, where dispersed fluorescence from excited electronic states can cover more than $5000 \mathrm{~cm}^{-1}$; further 
improvement was therefore imperative. In these situations, we fit the phase spectrum twice: first, to get the $\phi_{0}$ and $\beta$ parameters for the linear function, and again to get parameters $\phi_{0(2)}, \beta_{2}$ and $C_{2}$ for a quadratic fit to the function $\phi_{2}(\omega)=\phi_{0(2)}+\beta_{2} \omega+C_{2} \omega^{2}$. In Eq. 49 of Ref. [2], which gives the transformed spectrum $P_{A}(\omega)$ in terms of the interferogram function $\gamma(x)$ and an instrument response function $A$ as

$$
P_{A}(\omega)=\operatorname{Re}\left\{e^{i \phi(\omega)} \frac{2}{\pi} \int_{-\infty}^{\infty} d x \gamma(x) A(x-\beta) e^{-i \omega x}\right\}
$$

we retained the $\beta$ factor for the linear function inside the integral, but used the quadratic function $\phi_{2}(\omega)$ in place of $\phi(\omega)$ in the factor outside of the integral. This procedure is empirical, but had the desired effect of improving lineshapes in the final transformed spectrum.

The second approach is described by Mertz in Ref. [3]. It involves calculating a phase function at low resolution as described above, but with zeropadding to the same number of points as the (padded) full interferogram. A high-resolution phase function is then obtained from the full interferogram, and the difference between the two is used to phase-correct the final spectrum during transformation. This process takes more time than does Porter's method, since two large Fourier transforms need to be computed, but on modern computers this is not a significant problem, and the phase correction seems consistently better when we use the Mertz method. Other approaches are certainly possible, like the one described by Forman, Steel and Vanasse[4]. 


\subsection{Factors affecting signal-to-noise ratio}

A region of the laser-induced fluorescence spectrum of the the ${ }^{60} \mathrm{NiD}$ isotopologue of nickel deuteride is shown in Fig. 3, taken with comparable numbers of scans with both the PCDA and ACCES IO units. The spectra are completely comparable in quality, but an improvement in signal-to-noise ratio when the ACCES IO unit is used is evident. We have explored some possible reasons for this.

1. We sample all of the signals more frequently with the ACCES box than the PCDA system does, and interpolate to achieve 16 points per He-Ne fringe. If we instead interpolate our data set at 8 or 4 points per fringe, and then work out the RMS value of the spectrum in a region free of data like the central part shown in Fig. 3, we obtain relative values of 1:1.004:1.235. Thus, the change in going from 16 to 8 samples per fringe is slight, but the noise level increases when only 4 samples are taken. Perhaps part of the improvement with increasing sampling is due to better definition of the He-Ne fringe zero crossings, with relatively stable interpolation between them. The PCDA sampled 4 times per fringe in the spectrum shown, with every second sample triggered in coincidence with a zero crossing, and with the triggering of interleaved samples set by a predictive algorithm. The method employing the ACCES box loses the exactness of the hardware trigger in determining a zero crossing, but with a sufficiently high sampling rate the interpolated fringe positions seem to be quite accurate.

2. Data processing seems to make a difference, too. The PCDA interferogram was processed with Bomem's software, and was then processed 
again with our software based on the Mertz method. Relative to the spectrum obtained at 16 points/fringe from the point above, we obtained RMS levels of 1.477 (Bomem software) and 1.170 (our software).

We conclude that the differences in signal-to-noise ratio seen in Fig. 3 arise from differences in the sampling rate as well as from differences in the phase correction and transformation. Tests taken from LIF of $\mathrm{I}_{2}$ are shown in Fig. 4; in the top trace, the expanded bandwidth obtained with our method is shown, while in the bottom trace a comparison of signal-to-noise between the PCDA and DAQ2006 systems is given. The improvement in signal-tonoise ratio using the DAQ2006 system is less evident than demonstrated with the free-standing ACCES IO box, most likely due to corrections that had to be applied to the DAQ2006 data to account for crosstalk between $\mathrm{D} / \mathrm{A}$ channels, as well as to some high-frequency noise that we suspect to come from it being plugged into the computer's motherboard.

\section{Conclusions}

We have shown that Bomem DA3 instruments that are still functioning mechanically and electrically can continue to be used for high-resolution molecular spectroscopy by collecting and processing the data they provide with a modern computer and data acquisition system, with improvements to the signal-to-noise ratio over spectra collected with the original systems. The cost of implementation is relatively modest, about $\$ 800$ US for an ACCES IO unit or about 1200 Euros for the DAQ2006 card. The new data collection procedures generated files much larger than those created by the original computer systems, and require considerably more disk space to store, but 

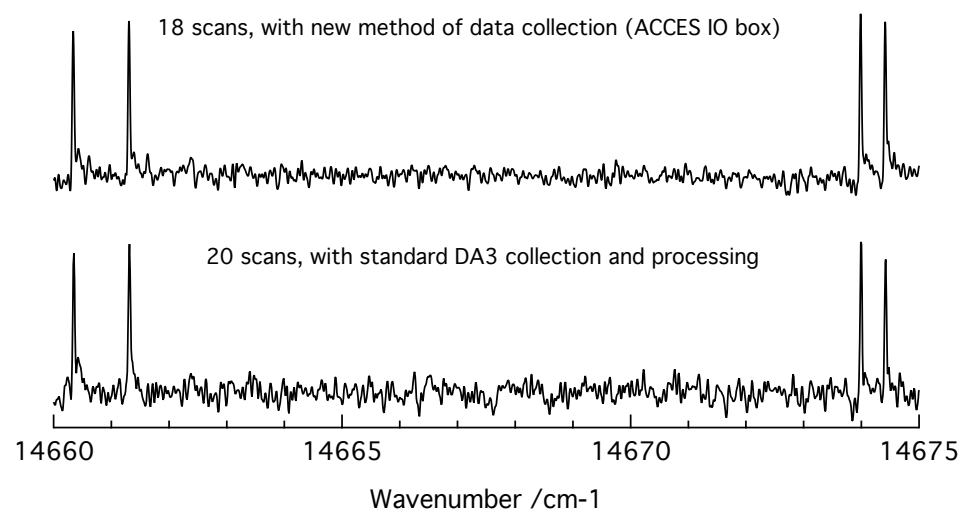

Figure 3: Spectra of ${ }^{60} \mathrm{NiD}$ collected and processed with the Bomem PCDA system (bottom, 20 scans coadded) and with the ACCES IO box and our own software (top, 18 scans). The signal-to-noise ratio in the bottom trace is about twice that of the top trace for a comparable number of scans.

the increased density of sampled points and the freedom to reject individual scans that might exhibit artifacts lead to improvements in the signal-to-noise ratio in the final interferograms. Some of these ideas have been explored previously in some fashion, including sampling the signals with more bit depth [5], increasing the sampling rate[6], and interpolation methods [7, 8].

We present an example illustrating the advantages of rejecting bad interferograms in Fig. 5. The four interferograms shown at the top of the figure are taken from laser-induced fluorescence of $\mathrm{I}_{2}$, but in scans 3 and 4 the laser exciting the LIF hops off resonance and then on again. These scans be ignored in post-processing when taken with the DAQ card, wheras the original DA3 software sums all scans regardless, producing a lower-quality spectrum with a higher number of scans. The effect of mode-hops in laser-induced fluorescence are far more dramatic if they occur close to zero path difference 

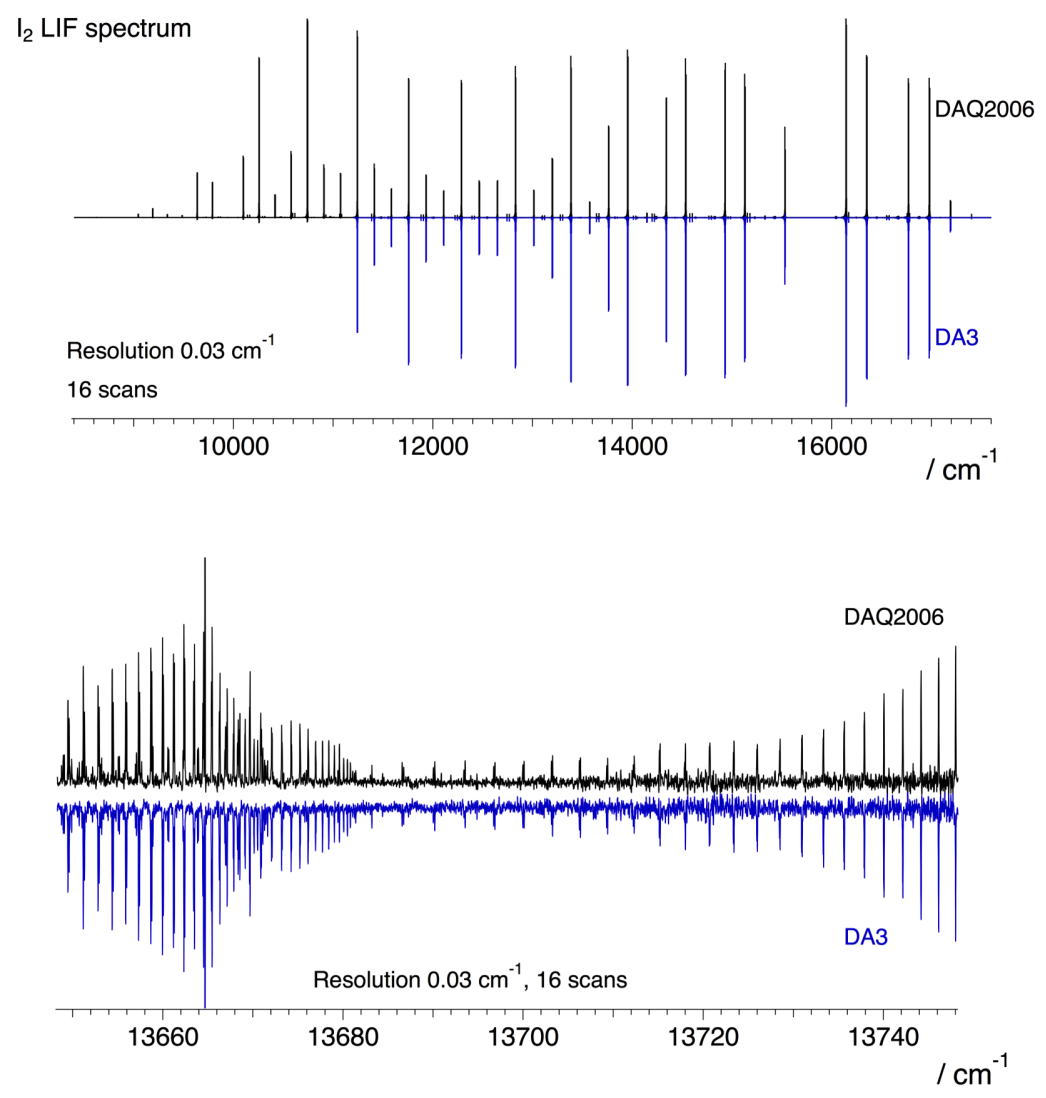

Figure 4: Laser-induced fluorescence spectra of $\mathrm{I}_{2}$ obtained with our methods (using the DAQ2006 A/D converter card) and with the PCDA system, taken simultaneously on the Lyon DA3. The top trace shows the expanded bandwidth available with our method, while the bottom spectrum shows the (slight) improvement in the signal-to-noise ratio when the our method is used. Note that the lower trace shows some of the weakest features in the spectrum, which result from rotational and vibrational relaxation of the upper level excited by the laser. 
as they do in interferogram 3; under such circumstances the final spectrum may be severely corrupted.

In the short time that original DA3 of Anthony Merer et al. has been operating at UNB, we have demonstrated the utility of the revived instrument for research by collecting new laser-induced fluorescence spectra of MgS and $\mathrm{FeH} / \mathrm{FeD}$ at high resolution. We envision future possible improvements as we begin to use this spectrometer in further experiments. We are considering replacing the assembly containing the single-mode He-Ne laser with an apparatus consisting of an optical fibre mount, a beam-expanding lens and a collimator. By injecting the reference laser through a fibre, we can exchange a failed He-Ne laser tube for a functioning one without disturbing the spectrometer alignment. A fibre injection system also allows us to conveniently use other single-mode laser wavelengths as the reference; for example, a diode laser locked to a rubidium resonance would provide a reference beam near $780 \mathrm{~nm}[9]$, quite advantageous if a spectrum near the He-Ne wavelength is desired but is unattainable due to simultaneous detection of scattered laser radiation at that wavelength. Improvements to the data collection system are also under consideration, particularly in the use of parallel processing in which one process monitors the serial communication between the computer and Bomem, another the collection and storage of digitized signal streams, while another provides real-time status of the scans as they come in. In this scenario, the last stream could extract and store only the point number at which a change of state in the ZPD signal occurs, so that storage of whole ZPD waveform would be unnecessary.

Finally we note that many of the procedures and processes that we have 

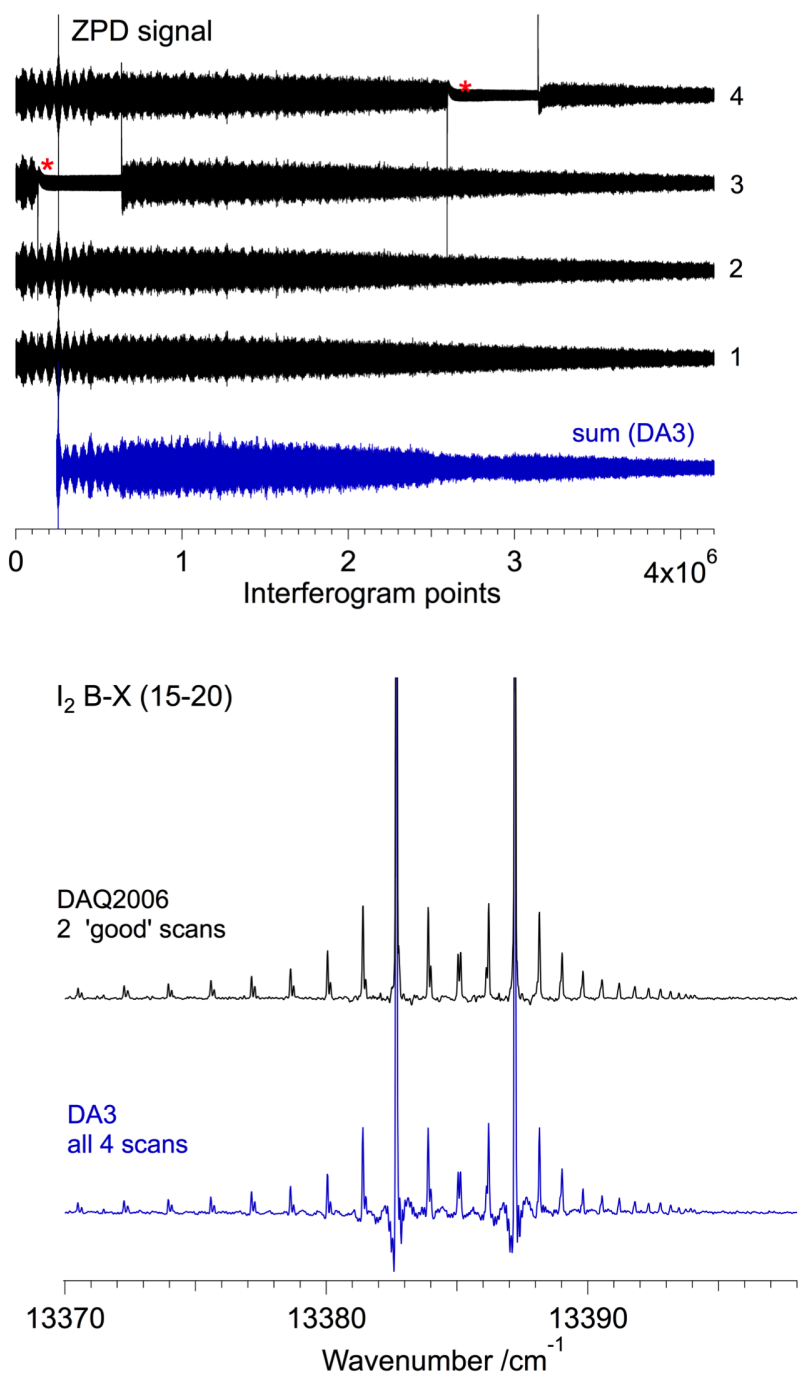

Figure 5: Being able to select which interferograms to include in summation allows corrupt data to be discarded. In this case, laser mode-hops (indicated with a red $*$ in interferograms 3 and 4) marred some scans of laser-induced fluorescence in molecular iodine. The top spectrum was produced by transforming the sum of only the 'good' interferograms 1 and 2 , while the bottom spectrum resulted from the PCDA system's transformation from the sum of all four. The distortions in lineshape and lower signal-to-noise ratio in the PCDA spectrum are evident. 
outlined here can be applied more generally than to just the Bomem DA3 spectrometers. The principles underlying our work might be applied to commercial FT spectrometers for which comparable documentation and access to signals is available, or to home-built FT instruments (for example, see Ref. $[10])$.

\section{Acknowledegments}

We are grateful to Joyce MacGregor and Brian Titus at UNB for technical assistance, to Chris Chan for initial assembly and alignment of the UBC spectrometer upon its arrival at UNB, to Dare Winters of ACCES IO for her expert assistance with programming the USB-AIO16-16E unit, and to Michael Eliades of ASEE Products, Inc for many insightful discussions. Financial support for this project was provided by the Natural Sciences and Engineering Research Council of Canada (NSERC) and le Centre national de la recherche scientifique (CNRS). DWT is grateful for support from the CNRS for a Chercheur Invité appointment at the Institut Lumière Matière

during his stay in Lyon, and AJR for support from the Harrison McCain Foundation for her 2017 visit to UNB. 


\section{References}

[1] D. J. W. Kendall, H. L. Buijs, J. W. C. Johns, J. Mol. Struct. 79 (1982) 39-42.

[2] C. D. Porter, D. B. Tanner, Int. J. Infrared Millimeter Waves 4 (1983) 273-298.

[3] L. Mertz, Infrared Physics 7 (1967) 17-23.

[4] M. L. Forman, W. H. Steel, G. A. Vanasse, J. Opt. Soc. Am. 56 (1966) 59-63.

[5] J. W. Brault, Appl. Opt. 35 (1996) 2891-2896.

[6] R. Panzeri, B. Saggin, D. Scaccabarozi, M. Tarabini, in: R. Meynart, S. P. Neeck, T. Kimura, H. Shimoda (Eds.), Sensors, Systems and NextGeneration Satellites XX, volume 10000 of Proceedings of the SPIE, Society of Photographic Instrumentation Engineers, SPIE, 2016, p. 100001F.

[7] L. Yan, L. Sheng, G. Minguang, X. Liang, L. Xiangxian, Infrared and Laser Engineering 47 (2015) 0123001.

[8] L. Yan, L. Sheng, G. Minguang, X. Liang, F. Mingchun, Y. Shubin, L. Jiangua, L. Xiangxian, Acta Optica Sinica 35 (2016) 0930005.

[9] C. P. Pearman, C. S. Adams, S. G. Cox, P. F. Griffin, D. Smith, I. G. Hughes, J. Phys. B: At., Mol. Opt. Phys. 35 (2002) 5141-5151.

[10] A. Libert, Design of a Fourier transform spectrometer for high resolution spectroscopy, Master's thesis, Université catholique de Louvain, 2016. 